\begin{tabular}{|c|c|}
\hline Title & Lack of association between X BP1 genotype and calcium signaling in the platelets of healthy subjects \\
\hline Author(s) & $\begin{array}{l}\text { Kusumi, Ichiro; Masui, Takuya; Kakiuchi, Chihiro; Suzuki, Katsuji; A kimoto, Tatsuyuki; Hashimoto, Ryota; Kunugi, } \\
\text { Hiroshi; Kato, T adafumi; Kayama, T sukasa }\end{array}$ \\
\hline Citation & $\begin{array}{l}\text { Neuroscience Letters, 369(1), 1-3 } \\
\text { https://doi.org/10.1016/.neulet.2004.07.003 }\end{array}$ \\
\hline Issue Date & 2004-10-07 \\
\hline Doc URL & http:/hdl .handle.net/2115/8433 \\
\hline Type & article (author version) \\
\hline Note(URL) & http://www.sciencedirect.com/sciencejournal 03043940 \\
\hline File Information & NSL04.pdf \\
\hline
\end{tabular}

Instructions for use 
submitted to Neuroscience Letters

2283 words, 10 text pages and 1 table

\section{Lack of association between XBP1 genotype and calcium signaling in the platelets of healthy subjects}

Ichiro Kusumi $^{1}$, Takuya Masui ${ }^{1}$, Chihiro Kakiuchi ${ }^{2}$, Katsuji Suzuki ${ }^{1}$, Tatsuyuki Akimoto ${ }^{1}$, Ryota Hashimoto ${ }^{3}$, Hiroshi Kunugi ${ }^{3}$, Tadafumi Kato ${ }^{2}$ and Tsukasa Koyama ${ }^{1}$

${ }^{1}$ Department of Psychiatry, Hokkaido University Graduate School of Medicine Sapporo, Japan

${ }^{2}$ Laboratory for Molecular Dynamics of Mental Disorders, Brain Science Institute, RIKEN, Wako, Japan

${ }^{3}$ Department of Mental Disorder Research, National Institute of Neuroscience, NCNP, Kodaira, Japan

Correspondence: Ichiro Kusumi, M.D., Ph.D.

Department of Psychiatry, Hokkaido University Graduate School of Medicine

North 15, West 7, Sapporo 060-8638, Japan

TEL: +81-11-716-1161 ext.5973 
FAX: +81-11-706-5081

e-mail: ikusumi@med.hokudai.ac.jp 


\begin{abstract}
Dysregulations of calcium (Ca) homeostasis may be involved in the pathophysiology of bipolar disorder. Enhanced Ca response to various agonists in peripheral blood cells is one of a few confirmed biological markers for bipolar disorder. Recently, a polymorphism of XBP1, a pivotal gene in the endoplasmic reticulum (ER) stress response, was shown to contribute to the genetic risk factor for bipolar disorder. Thus, in this study, we examined the relationship between the XBP1 gene polymorphism and the Ca signaling in the platelets of healthy controls. The present results suggest no significant difference in the basal Ca level or 5-HT-induced Ca mobilization among normal subjects with $-116 \mathrm{C} / \mathrm{C}, \mathrm{C} / \mathrm{G}$ and $\mathrm{G} / \mathrm{G}$ genotypes. Further investigations are necessary to examine the relationship in the different peripheral blood cells and/or in larger samples from patients with bipolar disorder.
\end{abstract}

Key words: Calcium; XBP1; Endoplamic reticulum; Bipolar disorder; Polymorphism; Healthy subject

Altered calcium (Ca) signaling has been reported in the peripheral blood cells of patients with bipolar disorder. We have reported that serotonin (5-HT)- or thrombininduced intracellular Ca mobilization is enhanced in the platelets of unmedicated patients with bipolar disorder [1-3]. Other researchers also indicated similar findings in platelets [4-7], in neutrophils [8], and in transformed lymphoblastoid cells [9]. 
Our longitudinal follow-up study suggested that the enhanced Ca response is trait dependent [1] and that patients with marked increased Ca response are good responders to mood stabilizers such as lithium and valproate [10]. These findings in non-neuronal cells, which could provide clues to the molecular basis of the disease, suggest that the altered Ca mobilization might be involved in the pathophysiology of bipolar disorder. The enhanced Ca response could be due to altered functioning of endoplasmic reticulum (ER) [9], mitochondria [11] or some other signal transduction pathways [12,13]. ER is the site of complex processes such as Ca storage, Ca signaling, processing and folding of newly synthesized membrane and secretory proteins, and triggering of cell response to severe forms of stress, which interfere with ER functions [14]. Cell injury may develop under conditions where ER Ca homeostasis and/or folding or processing of proteins is disturbed (referred to as ER stress), leading to the activation of the unfolded protein response such as suppression of protein synthesis and expression of ER stress-related genes including XBP1 and GRP78/BiP [14].

Recently, a polymorphism of the XBP1 gene was shown to contribute to the genetic risk factor for bipolar disorder [15]. The polymorphism $(-116 \mathrm{C} \rightarrow \mathrm{G})$ in the promoter region of XBP1 was significantly more common in Japanese bipolar patients (odds ratio=4.6) and overtransmitted to affected offspring in trio samples of the NIMH Bipolar Disorder Genetic Initiative [15]. XBP1-dependent transcription activity of the $-116 \mathrm{G}$ allele was lower than that of the $-116 \mathrm{C}$ allele, and in the cells with the G allele, induction of XBP1 expression after ER stress was markedly 
reduced [15]. Moreover, valproate rescued the impaired response by inducing ATF6, the gene upstream of XBP1 [15]. Darier's disease, an autosomal-dominant skin disorder, which is sometimes accompanied by bipolar disorder, was reported to be caused by mutations in the ER calcium adenosine triphosphatase (Ca-ATPase) gene [16]. In addition, the recent finding that valproate increases expression of the ER stress protein also suggests that the ER plays a role in bipolar disorder [17].

Therefore, it is possible that the altered Ca signaling may be due to the dysfunction of ER stress response by -116G allele of the XBP1 gene. In this study we examined the relationship between the $-116 \mathrm{C} / \mathrm{G}$ polymorphism of the XBP1 gene and the basal Ca level or 5-HT-induced Ca response in the platelets of healthy subjects.

One hundred and seventeen unrelated healthy volunteers were all Japanese recruited from laboratory, office or hospital staff at Hokkaido University. They all underwent a direct interview to exclude psychiatric disorders classified according to DSM-IV. There were 82 males and 35 females, and the average age was $34.1 \pm 8.7$ (mean \pm S.D.) years. They had no physical illness and were all drug free for at least 4 weeks before blood sampling. After complete description of the study, informed consent was obtained from all subjects. The research protocol was approved by the ethics committee of Hokkaido University Graduate School of Medicine.

The isolation of platelets and the measurement of intraplatelet Ca concentration were performed as described previously [18]. Briefly, platelet-rich plasma was incubated with 4 uM fura-2-AM (Dojindo, Kumamoto, Japan), a Ca sensitive 
fluorescent probe, for $15 \mathrm{~min}$ at $37^{\circ} \mathrm{C}$. After centrifugation, the resulting platelet pellet was suspended at $1 \times 10^{8}$ cells/ml in Krebs-Ringer HEPES buffer. The samples were prewarmed in a cuvette at $37^{\circ} \mathrm{C}$ for 4 min and then $10 \mathrm{uM} \mathrm{5-HT \text {was }}$ added to the incubation medium. Fluorescence was measured on a Hitachi F-2000 fluorometer with excitation at 340 and $380 \mathrm{~nm}$, and with emission at $510 \mathrm{~nm}$. We measured both basal Ca concentration and the maximum Ca increase (initial peak resting level) induced by 10uM of 5-HT.

DNA was extracted from $20 \mathrm{ml}$ of whole blood by standard methods. For the genotyping of XBP1 gene -116C/G polymorphism, the TaqMan 5'-exonuclease allelic discrimination assay was used. Primers and probes for TaqMan assay were as follows:

primers, XBP1-F, CTGTCACTCCGGATGGAAATAAGTC, and XBP1-R, ATCCCTGGCCAAAGGTACTTG, and probes, XBP1-C, VICCTCCCGCACGTAAC-MGB, and XBP1-G, FAM-TCCCGCAGGTAAC-MBG. Amplification was performed under the following conditions: $10 \mathrm{~min}$ at $95^{\circ} \mathrm{C}, 40$ cycles of 15 sec at $92^{\circ} \mathrm{C}$ and 1 min at $60^{\circ} \mathrm{C}$ in an GeneAmp PCR system 9700 thermocycler. Genotypes were determined using an ABI 7000 HT sequence detection system (Applied Biosystems, Foster City, CA, US).

The associations between the XBP1 gene polymorphism and, basal Ca level or 5HT-induced Ca response were assessed by one-way ANOVA.

The basal Ca concentration and 5-HT-stimulated Ca response sorted by XBP1 gene polymorphism are shown in Table 1. Observed genotype distributions were 
consistent with Hardy-Weinberg equilibrium. The distribution of the XBP1

genotypes in our sample was almost same as in another Japanese sample [15]. There was no significant difference in the basal Ca level or 5-HT-induced Ca mobilization among the healthy subjects with $-116 \mathrm{C} / \mathrm{C}, \mathrm{C} / \mathrm{G}$ and $\mathrm{G} / \mathrm{G}$ genotypes (Table 1).

The following findings expect the possible relationship between the XBP1 gene polymorphism and the Ca signaling abnormality. First, the enhanced Ca response to various agonists in peripheral blood cells is one of a few confirmed biological markers for bipolar disorder [1-9]. Second, the dysregulations of Ca homeostasis may be involved in the pathophysiology of bipolar disorder, from the fact that the dysinhibition of Ca mobilization in the presence of PKC inhibitor is observed in bipolar disorder [12] and that agonist-induced Ca responses are enhanced in the presence of myosin light chain kinase inhibitor, which are reversed by treatment with lithium [13]. Third, it is possible that the ER dysfunction may result in abnormal Ca homeostasis. The Ca response to thapsigargin, a ER $\mathrm{Ca}^{2+}$-ATPase inhibitor, are reported to be enhanced in bipolar patients compared to unipolar patients and normal controls [19]. Fourth, the DNA microarray analysis of lymphoblastoid cells derived from pairs of twins discordant with respect to bipolar disorder suggests that the XBP1 polymorphism may contribute to the genetic risk factor for the illness [15]. Last, the expression of GRP78/BiP, one of ER stress proteins, plays a direct and important role in Ca mobilization. It is reported that agonist-stimulated Ca response and thapsigargin-induced Ca release are enhanced in GRP78/BiP-transfected cells compared to control cells [20]. 
Unexpectedly, the present results did not indicate any significant relationship between XBP1 genotype and basal Ca concentration or 5-HT-induced Ca mobilization in the platelets of healthy subjects. Since drug-free samples are indispensable to measure intraplatelet Ca concentration [18], in this preliminary study we first examine the association between XBP1 gene polymorphism and Ca signaling in healthy controls. We have already indicated that the range of the 5-HTstimulated Ca response in normal subjects is widely distributed and fairly overlapped with that in bipolar disorder [1]. Moreover, depressed patients with a Ca response above $280 \%$ of basal which corresponds to the value of the mean + 2SD for normal controls, exhibit a good response to mood stabilizers [10]. Thus, the higher Ca response depressed patients show, the more probably their diagnosis may be bipolar disorder. This parameter seems to be a continuous marker from normal to bipolar disorder. On the other hand, the G allele of XBP1 gene, a risk allele for bipolar disorder, is also observed in normal controls, not only in bipolar disorder [15]. Therefore, it is of significance to examine the relationship between the XBP1 genotype and the Ca response in normal subjects. Further studies are necessary to examine the relationship between them in patients with bipolar disorder. Another limitation of this study is to measure Ca response in the mature platelets that lack a nucleus and do not have a part of ER stress response including the XBP1 loop. Using lymphocytes or lymphoblastoid cells as samples may result in the different finding from the present study. 
In conclusion, the present study suggests that the XBP1 gene polymorphism is not associated with the basal Ca level or 5-HT-stimulated Ca response in the platelets of healthy control subjects. Further investigations are needed to examine the relationship in the different peripheral blood cells and/or in larger samples from patients with bipolar disorder.

\section{Acknowledgments}

This work was partly supported by grants-in-aid for Soul and diseases-of-thenervous-system research (T. Koyama) from Japanese Ministry of Health, Labour and Welfare, and for Scientific Research No. 15591206 (I. Kusumi) from Japanese Ministry of Education, Culture, Sports, Science and Technology.

\section{References}

[1] I. Kusumi, T. Koyama, I. Yamashita, Serotonin-induced platelet intracellular calcium mobilization in depressed patients, Psychopharmacology, 113 (1994) 322-327.

[2] I. Kusumi, T. Koyama T, I. Yamashita, Thrombin-induced platelet calcium mobilization is enhanced in bipolar disorders, Biol. Psychiatry, 32 (1992) 731734.

[3] K. Suzuki, I. Kusumi, Y. Sasaki, K. Koyama, Serotonin-induced platelet intracellular calcium mobilization in various psychiatric disorders: is it specific to bipolar disorder ?, J. Affect. Disord. 64 (2001) 291-296. 
[4] S.L. Dubovsky, C. Lee, J. Christiano, J. Murphy, Elevated platelet intracellular calcium concentration in bipolar disorder, Biol. Psychiatry 29 (1991) 441-450.

[5] Y. Okamoto, A. Kagaya, H. Shinno, N. Motohashi, S. Yamawaki, Serotonininduced platelet calcium mobilization is enhanced in mania, Life Sci. 56 (1995) 327-332.

[6] M. Berk, W. Bodemer, T. Van Oudenhove, N. Butkow, The platelet intracellular calcium response to serotonin is augmented in bipolar manic and depressed patients, Hum Psychopharmacol Clin Exp 10 (1995) 189-193.

[7] S. Yamawaki, A. Kagaya, Y. Okamoto, M. Shimizu, A. Nishida, Y. Uchitomi, Enhanced calcium response to serotonin in platelets from patients with affective disorders, J. Psychiatry Neurosci. 21 (1996) 321-324.

[8] D. van Calker, U. Foerstner, M. Bohus, P. Gebicke-Haerter, H. Hecht, H-J. Wark, M. Berger, Increased sensitivity to agonist stimulation of the Ca2+ response in neutrophils of manic-depressive patients: effects of lithium therapy, Neuropsycho-biology 27 (1993) 180-183.

[9] T. Kato, M. Ishiwata, K. Mori, S. Washizuka, O. Tajima, T. Akiyama, N. Kato, Mechanisums of altered $\mathrm{Ca}^{2+}$ signaling in transformed lymphoblastoid cells from patients with bipolar disorderm, Int. J. Neuropsychopharmacology 6 (2003) 379389.

[10] I. Kusumi, K. Suzuki, Y. Sasaki, K. Kameda, T. Koyama, Treatment response in depressed patients with enhanced Ca mobilization stimulated by serotonin. Neuropsychopharmacology 23 (2000) 690-696. 
[11] T. Kato, N. Kato, Mitochondrial dysfunction in bipolar disorder, Bipolar Disord. 2 (2000) 180-190.

[12] K. Suzuki, I. Kusumi, T. Akimoto, Y. Sasaki, T. Koyama, Altered 5-HT-induced calcium response in the presence of staurosporine in blood platelets from bipolar disorder patients, Neuropsychopharmacology 28 (2003) 1210-1214.

[13] K. Suzuki, I. Kusumi, T. Akimoto, Y. Sasaki, T. Koyama, Effects of lithium and valproate on agonist-induced platelet intracellular calcium mobilization: relevance to myosin light chain kinase, Prog. Neuro-Psychopharmacol \& Biol. Psychiatry 28 (2004) 67-72.

[14] W. Paschen, A. Frandsen, Endoplasmic reticulum dysfunction- a common denominator for cell injury in acute and degenerative diseases of the brain, J. Neurochem. 79 (2001) 719-725.

[15] C. Kakiuchi, K. Iwamoto, M. Ishiwata, M. Bundo, T. Kasahara, I. Kusumi, T. Tsujita, Y. Okazaki, S. Nanko, H. Kunugi, T. Sasaki, F. Kato, Impaired feedback regulation of XBP1 as a genetic risk factor for bipolar disorder. Nature Genetics 35 (2003) 171-175.

[16] N.J.O. Jacobsen, I. Lyons, B. Hoogendoorn, S. Burge, P-Y. Kwok, M.C. O’Donovan, N. Craddock, M.J. Owen, ATP2A2 mutations in Darier's disease and their relationship to neuropsychiatric phenotypes, Hum. Mol. Genet. 8 (1999) 1631-1636. 
[17] C.D. Bown, J.F. Wang, L.T. Young, Increased expression of endoplasmic reticulum stress proteins following chronic valproate treatment of rat C6 glioma cells, Neuropharmacology 39 (2000) 2162-2169.

[18] I. Kusumi, T. Koyama, I. Yamashita, Effect of various factors on serotonininduced $\mathrm{Ca}^{2+}$ response in human platelets, Life Sci. 48 (1991) 2405-2412.

[19] C. Hough, S-J. Lu, C.L. Davis, D-M. Chuang, R.M. Post, Elevated basal and thapsigargin-stimulated intracellular calcium of platelets and lymphocytes from bipolar affective disorder patients measured by a fluorometric microassay. Biol. Psychiatry 46 (1999) 247-255.

[20] J-P. Lievremont, R. Rizzuto, L. Hendershot, J. Meldolesi, BiP, a major chaperone protein of the endoplasmic reticulum lumen, plays a direct and important role in the storage of the rapidly exchanging pool of $\mathrm{Ca}^{2+}$, J. Biol. Chem. 272 (1997) 30873-30879. 
Table 1. Basal Ca level and 5-HT-induced Ca response sorted by -116C/G polymorphism of XBP1 gene in Japanese healthy subjects

\begin{tabular}{ccccc}
\hline Ca signalling & \multicolumn{3}{c}{ Genotype } & ANOVA \\
\cline { 2 - 4 } & $\begin{array}{c}\mathrm{C} / \mathrm{C} \\
(\mathrm{N}=12)\end{array}$ & $\begin{array}{c}\mathrm{C} / \mathrm{G} \\
(\mathrm{N}=58)\end{array}$ & $\begin{array}{c}\mathrm{G} / \mathrm{G} \\
(\mathrm{N}=47)\end{array}$ & \\
\hline Basal Ca level & $63.0 \pm 7.8$ & $59.8 \pm 2.2$ & $61.4 \pm 2.0$ & $\mathrm{~F}=0.23, \mathrm{p}=0.79$ \\
5-HT-induced Ca response & $76.3 \pm 8.0$ & $88.5 \pm 4.4$ & $85.8 \pm 3.6$ & $\mathrm{~F}=0.84, \mathrm{p}=0.44$ \\
& & & & \\
\hline
\end{tabular}

Results are expressed as mean \pm SEM. 